\title{
Recent Portal Vein Thrombosis in Liver Cirrhosis: A Case of Success in Reversion of Portal Hypertension
}

\section{Thales Girardi May ${ }^{1}$, Felipe Fiorin Carmona ${ }^{1}$, Lígia Rodrigues Simonetti $^{1}$, Jéssica Araújo Amparado ${ }^{1}$, Luis Fernando Joaquim ${ }^{2}$ and Rafael Lima Kahwage ${ }^{2 *}$}

${ }^{1}$ Department of General Medicine, Hospital Beneficente Santa Casa de Misericórdia de Ribeirão Preto, São Paulo, Brazil

${ }^{2}$ Department of General Internal Medicine, Faculty of Medicine of Ribeirão

Preto, University of São Paulo, Ribeirão Preto, São Paulo, Brazil

*Corresponding Author: Rafael Lima Kahwage, Department of General Medicine, Hospital Beneficente Santa Casa de Misericórdia de Ribeirão Preto, Campos Elísios, Ribeirão Preto, SP, Brazil.
Received: September 04, 2020

Published: October 14, 2020

(C) All rights are reserved by Rafael Lima

Kahwage., et al.

\begin{abstract}
Portal vein thrombosis is an unusual complication in patients with liver cirrhosis. In the current research of its genesis, an underlying prothrombotic state and a combination of local endothelial factors, family and/or acquired inheritance and other thrombophilic factors are demonstrated. This condition is described as a major cause of portal hypertension, whether the patient has liver dysfunction or not.

Clinical and laboratorial diagnosis are complemented with imaging exams and are useful in the early diagnosis, since the treatment directly affects the morbidity and mortality of the disease.

We aimed to describe a case of a recent portal vein thrombosis concomitantly with hepatic cirrhosis and thrombophilia. Partial recanalization of the thrombus was achieved with anticoagulant treatment and consequent reversal of signs and symptoms of portal hypertension.
\end{abstract}

Keywords: Portal Vein Thrombosis; Cirrhosis; Recanalization; Thrombophilia; Anticoagulation

\section{Introduction}

Portal vein thrombosis (PVT) refers to a complete or partial obstruction of the blood flow in the portal vein due to the presence of a thrombus. It is a rare entity, described for the first time in 1869 by Balfour and Stewart. It can be related to hepatic cirrhosis, hepatocellular carcinoma, or to any hepatic disease.

\section{Pathogenesis}

PVT is related to a compensatory mechanism immediately after the venous obstruction. Initially, there is a vasodilation of the hepatic artery and after a few days, there is a neoformation of the vessels. The total process concludes in 3 to 5 weeks. After the blood vessel occlusion, there is a stimulus in the apoptosis of the hepatocytes and liver lobes in which a hypoperfusion occurs. Thus, there is a slow and progressive decrease in liver function [1].

\section{Etiology}

PVT is frequently multifactorial, related, approximately, $70 \%$ to local factors and $30 \%$ to systemic factors. Among the main risk factors are abdominal inflammatory processes such as appendicitis, diverticulitis, pancreatitis, cholecystitis, liver abscess or cholangitis. The neoplasms represent $21 \%$ to $24 \%$ of cases with thrombosis related to the direct vascular invasion, local compression and hypercoagulability [2]. 
Cirrhotic patients have a higher risk of developing the disease, with an ascending prevalence according to the hepatic disfunction, corresponding to $30 \%$ in liver transplant candidates and $10 \%$ to $40 \%$ in patients with hepatocellular carcinoma $[3,4]$.

Among the systemic factors are myeloproliferative diseases and prothrombotic conditions ranging from $40 \%$ to $60 \%$. Mutations in the V Leiden factor, deficiency in the $\mathrm{C}, \mathrm{S}$ and antithrombin III proteins are the main prothrombotic conditions. Other risk factors are contraception, pregnancy, nocturnal paroxysmal hemoglobinuria, genetic mutations, trauma and idiopathic, corresponding up to $50 \%$ of the cases [5].

Hypercoagulability, endothelial injury and slowed blood flow (Virchow triad) must be suspected in PVT patients. However, prothrombotic conditions should be considered and researched whenever possible.

\section{Classification}

Portal venous thrombosis classifications may vary in the literature. A novel classification in cirrhotic patients worked on the combination of different variants [6]. This is an anatomic-functional classification and considers:

1. Site of PVT (Type 1: only trunk. Type 2: only branch; 2a, one branch; $2 \mathrm{~b}$, both branches. Type 3: trunk and branches);

2. Degree of portal venous system occlusion (occlusive or nonocclusive; flow visible on doppler ultrasound);

3. Duration (recent - first time detected in a previously patent portal vein with hyperdense thrombus, no collateral, dilated portal vein and no cavernoma - or chronic) and presentation (asymptomatic or symptomatic);

4. Extent of portal venous system occlusion (splenic, mesenteric or both);

5. Type and presence of underlying disease (cirrhotic, non-cirrhotic, post-liver transplant, hepatocellular carcinoma, local malignancies and associated conditions).

\section{Clinical, laboratory and imaging features}

The clinical presentation depends on the time of the appearance of the disease, the extent of the affected container and the presence of collateral circulation. PVT can be associated to high mortality due to the hepatic ischemia, though there is low risk of bleeding in the varicose veins.
Patients with recent PVT can present abdominal pain or distention, rectal bleeding, nausea, vomit, anorexia, fever, metabolic acidosis, or sepsis. Most patients present splenomegaly, while the presence of ascites is a rare condition. Instead, chronic PVT curses with no symptoms, except for the formation of collateral circulation and ascites. The clinical suspicion is based on the incidental finding of hypersplenism, signs of hypertension or gastrointestinal bleeding.

Laboratorial diagnosis in PVT patients without hepatic disease associated is usually normal. However, markers of thrombogenic activity, as prothrombin and other coagulation factors, show themselves moderately decreased [7].

Abdominal ultrasonography is a low-cost exam and shows high sensibility and specificity. A high probability diagnosis of PVT occurs when solid and echogenic material is evidenced inside the blood vessel.

Computerized tomography and/or magnetic resonance imaging (MRI) are useful to evaluate the extension of the thrombus, presence of mesenteric and collateral circulation, involvement of adjacent organs. These exams also enable the characterization of neoplastic nodules sometimes associated with PVT.

\section{Treatment}

Although it has already been described the spontaneous resolution of PVT in the recent phase, the specific therapeutic handling is mandatory to solve the portal obstruction and avoid complications, mainly to prevent the progression to a chronic phase [8].

The main objectives of the treatment are the correction of risk factors, the prevention of the extension of thrombosis and the attainment of portal vein permeability [5].

Anticoagulant therapy is the main therapeutic approach to obtain total or partial venous recanalization in PVT patients not related to neoplasms. Different treatment classes are available: unfractionated heparin, low-molecular-weight heparin, vitamin $\mathrm{K}$ antagonists, direct-acting oral anticoagulants.

The recent PVT obtains a complete venous recanalization after 6 months of anticoagulant therapy in approximately $50 \%$ of the cases with low complication rates. However, in approximately $10 \%$ of the cases, the thrombosis is refractory to therapy [9]. In addition, when a gastrointestinal infarction occurs, the anticoagulants

Citation: Rafael Lima Kahwage., et al. "Recent Portal Vein Thrombosis in Liver Cirrhosis: A Case of Success in Reversion of Portal Hypertension”. Acta Scientific Gastrointestinal Disorders 3.11 (2020): 13-19. 
administered before surgery appear to have a considerable survival benefit. The earlier the treatment starts, the better the clinical results are. Recent PVT curses with complete recanalization up to $69 \%$ when anticoagulation is instituted within the first week of diagnosis, reducing to less than $25 \%$ if initiated posteriorly [10].

Therapeutic options and timing in chronic thrombosis are controversial. Anticoagulant therapy is administrated in only $30 \%$ of the patients with PVT, reflecting concern about the presence of bleeding from esophageal varices, thrombocytopenia or coagulation disorders [1]. Nevertheless, patients receiving anticoagulant therapy have a low incidence of bleeding, and after follow-up studies, anticoagulant therapy has been shown to be effective in preventing new thrombotic events with low mortality [8].

There is no consensus on anticoagulant therapy use in cirrhotic patients. There is a high tendency to choose anticoagulants in the recent phase of the disease. Indication becomes controversial in chronic PVT. In this situation, adverse outcomes like hemorrhage from esophageal varices is customary [11]. Other authors recommend continued anticoagulant therapy only in patients with confirmation of thrombotic disorders or family history of venous thrombosis [12].

Long-term use of anticoagulants in PVT cirrhotic patients are not encouraged until their safety and efficacy have been proven. Signs of intestinal ischemia or infarct, in addition to the subjacent prothrombotic disorder, are relevant to the clinical context and should be considered as an indication for anticoagulant therapy in patients with liver cirrhosis. Still, their use should only occur after appropriate prophylaxis for bleeding from esophageal or gastric varices has been established $[1,13]$.

In patients without cirrhosis or neoplasms, PVT generally has good therapeutic results. However, the prognosis depends on the base conditions of the patient. A common complication is gastrointestinal hemorrhage associated to portal hypertension and more rarely, bile complications. Ascites, bacterial infection and hepatic encephalopathy are rare, except in the subsequence of gastrointestinal hemorrhage events.

In recent PVT without thrombophilic disorder, anticoagulation must be administered from 3 to 6 months continuously until complete venous recanalization can be evidenced [1]. Specialists recommend the use of anticoagulant therapy only in patients with a known diagnosis of thrombophilia or family history of venous thrombosis. These indications are correlated with improvement in survival and in reducing gastrointestinal hemorrhage [12]. In documented cases of thrombophilia, anticoagulation must be maintained for undetermined time.

Other therapeutic modalities are feasible in cases of absence of recanalization or partial thrombosis resolution after anticoagulant therapy. Different therapeutic options are possible, but they are still quite controversial and include thrombolysis, surgical thrombectomy, TIPS (transjugular intrahepatic portosystemic shunt), and surgical shunt (distal splenorenal shunt) [8,11].

Surgical thrombectomy is normally not recommended due to the high risks of morbidity and mortality. Percutaneous transhepatic mechanical thrombectomy can also be effective in recent thrombosis, but vascular trauma is common and can stimulate the recurrence of thrombosis posteriorly [14].

Another possible therapeutic approach is establishing a transjugular intrahepatic portosystemic anastomosis. This therapeutic option has its indication reserved to patients that develop recent PVT before or after hepatic transplant or in alternative to thrombolysis when anticoagulation fails [15].

Portal venous thrombosis is a factor of poor prognosis when present in patients with liver cirrhosis. It is an independent mortality factor both in pre and postoperative liver transplantation [16]. Postoperative complications are more important in patients with PVT and related to renal insufficiency, primary graft non-functioning and re-thrombosis [17]. Another study also showed that PVT adds important difficulties in liver transplantation, such as longer time of surgery, greater need for transfusion, higher incidence of renal insufficiency and re-thrombosis and need for complex surgical techniques [18].

We aimed to present a case report of a type 1, occlusive, recent portal venous thrombosis (PVT) in a patient with liver cirrhosis and a coagulation imbalance with satisfactory evolution. Active clinical investigations and therapeutic approaches were discussed.

\section{Case Report}

S.C., male, 61 years old, Caucasian, married, born in São Simão (a city in the interior of the state of São Paulo), from Ribeirão Preto, a truck driver. 
He was admitted to the general practice ward of Santa Casa of Ribeirao Preto, on 11/20/2018, with the report of a worsening in the general state and oscillation of the level of consciousness in the past, one day before the admission.

History of the current disease was related to a recent hospitalization in the same service due to upper gastrointestinal bleeding (UGIB) related to peptic ulcer disease classified as Forrest III. He had previous diagnosis of hepatic cirrhosis of alcoholic etiology Child B and Meld 16.

Physical examination at admission presented as regular general condition, icteric 1+/4+, Glasgow 12 (E3/V4/M5), presenting episodes of mental confusion, absent flapping, globose abdomen, palpable liver $5 \mathrm{~cm}$ from the costal margin.

The personal precedent is of noteworthy: diabetes type 2, risky alcoholic consumption (daily alcoholism with consumption of distillates above 30 grams per day) and smoking for 40 years. He presented recent hospitalization due to the UGIB (as described above) and hepatic encephalopathy grade II.

Family history has been made with an extensive research and there were no reports of thromboembolic phenomena or any hematologic diseases.

Complementary exams on 11/20/2018 evidenced: hemoglobin of $11.60 \mathrm{~g} / \mathrm{dL}$ (RV 13.0 to $18.0 \mathrm{~g} / \mathrm{dL}$ ); hematocrit of $34.40 \%$ (RV 42 to $53 \%$ ); leukocytes of 3.900 cells $/ \mathrm{mm}^{3}$ (RV 3.5 to $11.000 / \mathrm{mm}^{3}$ ); platelets count of $95.000 / \mathrm{mm}^{3}$ (RV 140.000 to $450.000 / \mathrm{mm}^{3}$ ); Activated partial thromboplastin time of $30.3 \mathrm{sec}$. (RV 22.7 to 31.8 sec.); INR of 1.25 (RV up to 1.2); Total bilirubin of $1.40 \mathrm{mg} / \mathrm{dL}$ (RV 0.00 to $1.20 \mathrm{mg} / \mathrm{dL}$ ); Direct bilirubin of $1.10 \mathrm{mg} / \mathrm{dl}$ (RV 0.00 to 0.40 $\mathrm{mg} / \mathrm{dL}$ ); Total proteins of $6.50 \mathrm{~g} / \mathrm{dL}$ (RV 6.4 to $8.3 \mathrm{~g} / \mathrm{dL}$ ); Albumin of $3.40 \mathrm{~g} / \mathrm{dl}$ (RV 3.5 to $5.2 \mathrm{~g} / \mathrm{dL}$ ); Oxaloacetic Transaminase (GOT/ SGOT) of $34 \mathrm{U} / \mathrm{L}(\mathrm{RV}<40 \mathrm{U} / \mathrm{L})$; Pyruvic Transaminase of $35 \mathrm{U} / \mathrm{L}$ (GPT/ALT) $(\mathrm{RV}<45 \mathrm{U} / \mathrm{L})$.

Abdominal ultrasonography on 11/22/2018 evidenced signs of chronic hepatopathy and portal vein thrombosis (Figure 1).

A previous doppler ultrasound image, performed at the Radiology Service of Santa Casa of Ribeirao Preto, on 10/17/2018, showed signs of chronic liver disease, but with normal distribution and caliber of the hepatic and portal veins.

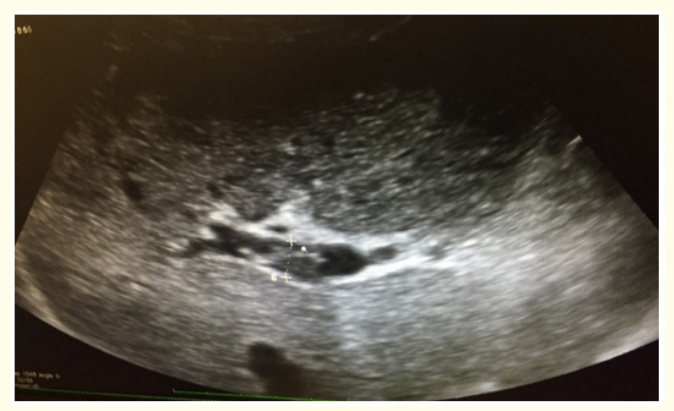

Figure 1: Ultrasonography of upper abdomen shows portal vein with $1.1 \mathrm{~cm}$ diameter, echogenic material without flow in doppler study and change in the hepatic echotexture.

In addition, an endoscopic image of 10/11/2018 showed esophageal varices of thin and medium caliber in the distal esophagus (Figure 2).

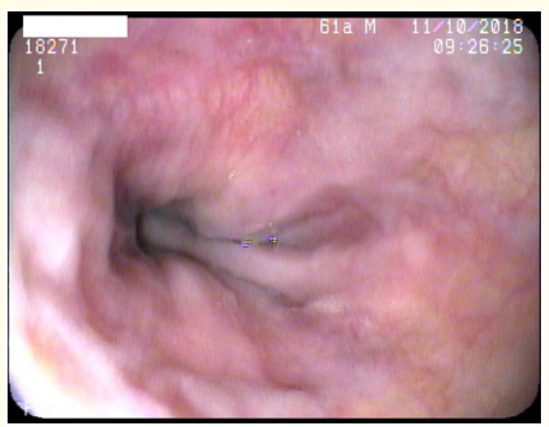

Figure 2: Upper digestive endoscopy showing bluish, tortuous varices, with no signs of red color in distal esophagus.

Therefore, therapeutic anticoagulation with enoxaparin sodium at $1 \mathrm{mg} / \mathrm{kg}$ for 12/12 hours was started. Seven days after the beginning of the described therapy and clinical compensation of the patient's condition, the patient underwent a new upper digestive endoscopy. An elastic bandage was performed in order to provide the eradication of esophageal varices and, therefore, maintain therapeutic safety in his anticoagulation. However, the exam showed only incipient varices in the distal esophagus (Figure 3).

Finally, at the end of the 7-day treatment with enoxaparin, a magnetic resonance scan of the abdomen was performed on $11 / 27 / 2018$, which showed the migration of the thrombus to the VI hepatic segment. 


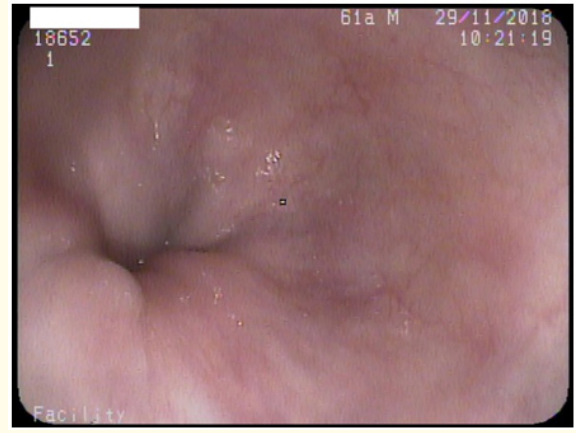

Figure 3: Upper digestive endoscopy demonstrating incipient varices in the distal esophagus, along the esophageal-gastric transition.

Other complementary exams were requested with the following results: Partial Activated Thromboplastin Time of $38.5 \mathrm{sec}$ (RV 22.7 to $31.8 \mathrm{sec}$ ); Prothrombin Time of $15.2 \mathrm{sec}$ (RV 10.4 to 12.6 sec); INR of 1.43 (RV until 1.2); Anti-thrombin III 60\% (RV 75\% to $120 \%$ ); Alpha fetoprotein of $2.00 \mathrm{ng} / \mathrm{mL}$ (RV inferior to $7.0 \mathrm{ng} /$ $\mathrm{mL}$ ); CA $19-9$ of $43.9 \mathrm{U} / \mathrm{mL}$ (RV inferior to $39.0 \mathrm{U} / \mathrm{mL}$ ); Antibody anti-cardiolipin IgG inferior to $1 \mathrm{GPL}$ U/mL (RV Inferior 15 GPL U/ $\mathrm{mL}$ ); Antibody anti-cardiolipin IgM of 3.9 MPL U/mL (RV Inferior 12,5 MPL U/mL); absence of mutation in the V Leiden Factor and absence of mutation of the prothrombin (Factor II); Factor VIII on $154 \%$ (RV 50\% to 150\%); absence of antibody lupus anticoagulant; Protein S functional on 17\% (VR 60\% to 120\%); Protein S free antigenic on $27 \%$ (VR 87 to $130 \%$ ).

The patient was discharged with follow-up at the cardiologyanticoagulation ambulatory to continue treatment with warfarin. In the first 30-day period, patient was asymptomatic, and INR was aimed at a therapeutic interval from 2.0 to 3.0. The duration of treatment with warfarin was programmed for 6 months. The patient was waiting for an appointment in the hepatology outpatient clinic.

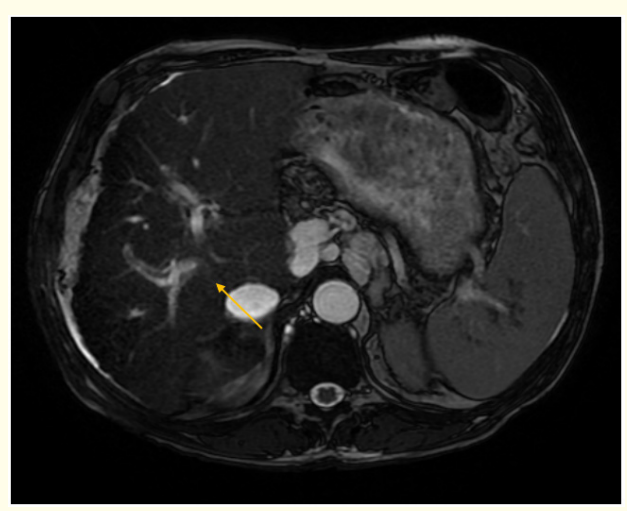

Figure 4: MRI of the abdomen evidencing the migration of the thrombos to the hepatic segment 6 (yellow arrow) and signs of chronic liver disease.

\section{Discussion}

Investigation of PVT in cirrhotic patient with portal hypertension and esophageal varices is applicable and necessary due to its high rate of incidence in this group of patients. PVT has an annual incidence of $10-15 \%$ in patients with liver cirrhosis. One reason is that the blood flow through the liver is slow in this condition and in the presence of several prothrombotic conditions [19]. Besides a considerable prevalence, cirrhotic patients with PVT have potential complications such as: progression to the chronic stage, portal hypertension with ascites, gastroesophageal varices, splenomegaly and intestinal necrosis. The diagnosis is made with an abdominal doppler ultrasound examination with good availability and low cost. The exam reveals the presence of solid and hyperechoic material in the portal vein, distension of the portal vein and/or tributary, and a net of collateral vases or cavernoma. In the present case, diagnosis was made, incidentally, with abdominal ultrasonography due to an investigation of hepatocellular carcinoma.

The etiological risks in our case were liver cirrhosis and thrombophilia related to functional and free protein S deficiency and Antithrombin III. A limitation of the present case was the unavailability of the protein $\mathrm{C}$ dosage by the laboratory panel at our center. In a recent study, about $50-60 \%$ of PVT cases presented polycythemia vera, essential thrombocythemia, primary myelofibrosis, mutation in prothrombin gene G20210A and antiphospholipid syndrome none of those conditions were diagnosed in our case [19].

There is little experience in management patients with cirrothic PVT. A recent publication proposed to start treatment in patients with liver cirrhosis, with recent PVT or with the presence of symptoms which are candidates to liver transplantation. Patients without this formal indication lack in evidence with a treatment of choice. Though, evidence show that patients with liver cirrhosis not eligible for liver transplantation may benefit from early PV recanalization with anticoagulants [19].

Interventional treatment as TIPS are reserved to prolong survival in clients with liver cirrhosis listed for hepatic transplantation. There are no studies with the use of thrombolytics in a liver cirrhosis group and this treatment should be avoided until new evidence is published.

In our case, we chose a 7-day treatment regimen with enoxaparin because of the availability, low cost and good evidence of expert consensus. Direct-acting oral anticoagulants are expensive and not available in our service and vitamin $\mathrm{K}$ antagonists have a limitation of prolongation of the prothrombin time in liver cirrhosis and also is a slow-acting drug.

After a 7-day treatment regimen with enoxaparin, our case presented good resolution of signs and symptoms with portal vein reperfusion and migration of thrombus to sixth hepatic segment, 
decreasing the risk of varicose hemorrhage and hepatic insufficiency [20]. Despite a significant number of published complications (gastrointestinal, genitourinary and central nervous system hemorrhages) [21] our patient presented no complications during treatment and after a 30-day follow-up.

\section{Conclusion}

We reported a type 1, occlusive, recent portal venous thrombosis associated with liver cirrhosis and thrombophilia (deficiency of functional and free protein S and Antithrombin III). After therapeutic anticoagulation with sodium enoxaparin, there was partial thrombus resolution with distal thrombus migration. The patient evolved with resolution of the portal hypertension and regression of the esophageal varices, thus deviating the risk of unfavorable evolution of these varices, related to a possible digestive bleeding. Therefore, early treatment prevented the progression of the disease to a chronic phase, which would imply a higher risk of complications from portal hypertension in a non-candidate for liver transplantation.

Screening, early diagnosis, investigation of prothrombotic factors (e.g.: mutations in factor V, factor II, factor VIII, deficiency in protein $\mathrm{C}, \mathrm{S}$ and antithrombin III) and treatment on a recent phase must be carefully evaluated in cirrhotic patients. Such factors may be crucial to the resolution of portal venous thrombosis and are related to increased patient survival.

\section{Conflicts of Interest}

The authors disclose no conflicts of interest.

\section{Writing Assistance}

Thales May, Felipe Carmona, Laís Simonetti, Jéssica Amparado, Rafael Lima Kahwage.

\section{Authors Contribution}

Thales Girardi May - Study concept and design; Analysis and interpretation of data; Critical revision of the manuscript; Translation and imaging work; Financial support.

Felipe Fiorin Carmona - Study concept and design; Analysis and interpretation of data; Critical revision of the manuscript; Financial support.

Lígia Rodrigues Simonetti - Study concept and design; Analysis and interpretation of data; Critical revision of the manuscript; Financial support.
Jéssica Araújo Amparado - Task organization; Study concept and design; Analysis and interpretation of data; Critical revision of the manuscript; Translation and imaging work; Financial support.

Luis Fernando Joaquim - Analysis and interpretation of data; Critical revision of the manuscript; Intellectual support; Financial support; Drafting of the manuscript; Administrative and technical support; Study supervision.

Rafael Lima Kahwage - Analysis and interpretation of data; Critical revision of the manuscript; Intellectual support; Financial support; Drafting of the manuscript; Administrative and technical support; Study supervision.

\section{Research Ethics Committee Statement}

The study was approved by the Research Ethics Committee (REC) of the Hospital Beneficente Santa Casa de Misericordia de Ribeirão Preto.

\section{Informed Consent Statement}

The patient participated in the study after becoming aware of and understanding the objectives, voluntarily agreeing to participate and signing the Free and Informed Consent Form.

\section{Bibliography}

1. Webster GJ., et al. "Review article: portal vein thrombosis new insights into aetiology and management". Alimentary Pharmacology and Therapeutics 21 (2005): 1-9.

2. Wang JT., et al. "Portal vein thrombosis". Hepatobiliary and Pancreatic Diseases International 4 (2005): 515-518.

3. Condat B and Valla D. "Nonmalignant portal vein thrombosis in adults". Nature Clinical Practice Gastroenterology and Hepatology 3 (2006): 505-515.

4. Ogren M., et al. "Trousseau's syndrome - what is the evidence? A population-based autopsy study". Thrombosis and Haemostasis 95 (2006): 541-545.

5. Ponziani FR., et al. "Portal vein thrombosis: insight into physiopathology, diagnosis, and treatment". World Journal of Gastroenterology 16.2 (2010):14355.

6. Sarin SK., et al. "Toward acomprehensive new classification of portal vein thrombosis in patients with cirrhosis". Gastroenterology 151 (2016): 574-577. 
7. Kocher G and Himmelmann A. "Portal vein thrombosis (PVT): a study of 20 non-cirrhotic cases". Swiss Medical Weekly 135 (2005): 372-376.

8. Simão A., et al. "Trombose da veia porta: importância do diagnóstico e tratamento precoces". Liver Today: Boletim Informativo APEF 7 (2009): 4-5.

9. Plessier A., et al. "A prospective multicentric follow-up study on 105 patients with acute portal vein thrombosis (PVT): Results from the european network for vascular disorders of the liver (en-vie)". Hepatology 46 (2007): 310.

10. Hoekstra J and Janssen HL. "Vascular liver disorders (II): portal vein thrombosis". The Netherlands Journal of Medicine 67 (2009): 46-53.

11. Boyer TD. "Management of portal vein thrombosis". Clinical Gastroenterology and Hepatology 4.10 (2008): 699-700.

12. Garcia-Pagán JC., et al. "Extrahepatic portal vein thrombosis". Seminars in Liver Disease 28 (2008): 282-292.

13. Amitrano L., et al. "Risk factors and clinical presentation of portal vein thrombosis in patients with liver cirrhosis". Journal of Hepatology 40 (2004): 736-741.

14. Rosendaal FR. "Venous thrombosis: a multicausal disease". Lancet 353 (1999): 1167-1173.

15. Ciccarelli 0., et al. "Transjugular intrahepatic portosystemic shunt approach and local thrombolysis for treatment of early posttransplant portal vein thrombosis". Transplantation 72 (2001): 159-161.

16. Tripodi A., et al. "Hypercoagulability in cirrhosis: causes and consequences". Journal of Thrombosis and Haemostasis 9 (2011): 1713-1723.

17. Egawa H., et al. "Single center experience of 39 patients with preoperative portal vein thrombosis among 404 adult living donor liver transplantations". Liver Transplantation 12 (2006): 1512-1518.

18. Englesbe MJ., et al. "Portal vein thrombosis and survival in patients with cirrhosis". Liver Transplantation 16.1 (2010): 8390.

19. Intagliata NM., et al. "Diagnosis, Development, and Treatment of Portal Vein Thrombosis in Patients With and Without Cirrhosis". Gastroenterology 156.6 (2019): 1582-1599.
20. Villa E., et al. "Enoxaparin prevents portal vein thrombosis and liver decompensation in patients with advanced cirrhosis". Gastroenterology 143 (2012): 1253-1260.

21. La Mura V., et al. "Harmful and beneficial effects of anticoagulants in patients with cirrhosis and portal vein thrombosis". Clinical Gastroenterology and Hepatology 16 (2018): 11461152.

\section{Assets from publication with us}

- Prompt Acknowledgement after receiving the article

- Thorough Double blinded peer review

- Rapid Publication

- Issue of Publication Certificate

- High visibility of your Published work

Website: www.actascientific.com/

Submit Article: www.actascientific.com/submission.php

Email us: editor@actascientific.com

Contact uS: +919182824667 